\title{
Supergravity and M-theory
}

\author{
Bernard de Wit • Maaike van Zalk
}

Received: 5 December 2008 / Accepted: 17 December 2008 / Published online: 10 February 2009

(C) The Author(s) 2009. This article is published with open access at Springerlink.com

\begin{abstract}
Supergravity provides the effective field theories for string compactifications. The deformation of the maximal supergravities by non-abelian gauge interactions is only possible for a restricted class of charges. Generically these 'gaugings' involve a hierarchy of $p$-form fields which belong to specific representations of the duality group. The group-theoretical structure of this $p$-form hierarchy exhibits many interesting features. In the case of maximal supergravity the class of allowed deformations has intriguing connections with $\mathrm{M} /$ string theory.
\end{abstract}

Keywords Supergravity · Gauging $\cdot$ M-theory

\section{Introduction}

Supergravity provides the effective field theories associated with string compactifications and serves as a framework for studying a large variety of phenomena. Among those are topics that have their roots in general relativity, such as black holes and cosmology. Irrespective of the precise context, supergravity itself leads to many surprises, which, in hindsight, often have an explanation in underlying theories, such as

This study is based on a talk presented at Quantum gravity: challenges and perspectives, Heraeus Seminar, Bad Honnef, 14-16 April 2008.

B. de Wit $(\bowtie) \cdot$ M. van Zalk

Institute for Theoretical Physics and Spinoza Institute, Utrecht University,

P. O. Box 80.195, 3508 TD, Utrecht, The Netherlands

e-mail: b.dewit@uu.nl

M. van Zalk

e-mail:m.vanzalk@uu.nl 
M-theory (an extension of string theory). Obviously this connection is at least partly based on the presence of non-trivial symmetries that are shared by these theories.

Here we discuss the deformations of (maximal) supergravities by non-abelian gauge interactions and exhibit some of their connections to M-theory. As it turns out, these deformations are quite restricted and can be classified by group-theoretical methods. They involve a hierarchy of $p$-form tensor fields, whose representations under the supergravity duality group have also been obtained from M-theory in various incarnations. The study of general gaugings of maximal supergravities, which was initiated in $[1,2]$, led to considerable insight in the general question of embedding a non-abelian gauge group into the rigid symmetry group $\mathrm{G}$ of a theory that contains abelian vector fields without corresponding charges, transforming in some representation of $\mathrm{G}$ (usually not in the adjoint representation). The field content of this theory is fixed up to possible (Hodge) dualities between $p$-forms and $(d-p-2)$-forms, so that it is advantageous to adopt a framework in which the decomposition of the form fields is left open until after specifying the gauging.

The relevance of this approach can, for instance, be seen in four space-time dimensions [3], where the Lagrangian can be changed by electric/magnetic duality so that electric gauge fields are replaced by their magnetic duals. In the usual setting, one has to adopt an electric/magnetic duality frame where the gauge fields associated with the desired gauging are all electric. In principle this may not suffice, as the gauge fields should also decompose under the embedded gauge group into fields transforming in the adjoint representation of the gauge group, and fields that are invariant under this group, so as to avoid inconsistencies. In a more covariant framework, on the other hand, one introduces both electric and magnetic gauge fields from the start, such that the desired gauge group can be embedded irrespectively of the particular electric/magnetic duality frame. Gauge charges can then be switched on in a fully covariant setting. Among other things this involves introducing 2-form fields transforming in the adjoint representation of $\mathrm{G}$. The gauge transformations associated with the 2-form gauge fields ensure that the number of physical degrees of freedom is not changed.

In this covariant approach the gauge group embedding is encoded in the so-called embedding tensor, which is treated as a spurionic quantity so as to make it amenable to group-theoretical methods. This embedding tensor was first introduced in the context of gaugings of three-dimensional maximal supergravity [4,5]. While every choice of embedding tensor defines a particular gauging and thereby a corresponding $p$-form hierarchy, scanning through all possible choices of the embedding tensor subject to certain group-theoretical representation constraints that it must obey, enables one to characterize the multiplicity of the various $p$-forms in entire G-representations-within which every specific gauging selects its proper subset. This is precisely the meaning of treating the embedding tensor as a spurionic quantity.

In four space-time dimensions no $p$-form fields are required in the action beyond $p=2$, but the higher-dimensional case naturally incorporates higher-rank form fields when switching on gauge charges, thus extending naturally to a hierarchy with a nontrivial entanglement of forms of different ranks. It may seem that one introduces an infinite number of degrees of freedom in this way, but, as mentioned already above, the hierarchy contains additional gauge invariances beyond those associated with the vector fields. This $p$-form hierarchy is entirely determined by the rigid symmetry group $\mathrm{G}$ 
and the embedding tensor that defines the gauge group embedding into $G[2,6]$ and a priori makes no reference to an action nor to the number $d$ of space-time dimensions. As a group-theoretical construct, the $p$-form hierarchy continues indefinitely, but in practice it can be consistently truncated in agreement with the space-time properties (notably the absence of forms of a rank $p>d$ ).

In the context of a given Lagrangian the details of the $p$-form hierarchy will change and the transformation rules are deformed by the presence of various matter fields. As a result, the closure of the generalized gauge algebra may involve additional symmetries. The hierarchy may turn out to be truncated at a relatively early stage, because the Lagrangian may be such that the gauge transformations that connect to the higher- $p$ forms have become trivially satisfied. On the other hand, the $(d-1)$ - and $d$-forms play a different role, as was suggested in [6], where this was explicitly demonstrated for three-dimensional maximal supergravity.

In this paper we review a number of elements of the $p$-form hierarchy and its connections to M-theory. In Sect. 2 we give a qualitative introduction to the so-called hidden symmetries that emerge in torus compactifications of higher-dimensional gravity theories, to appreciate some of the duality symmetries that are relevant for the maximal supergravities. In a separate Sect. 2.2, we demonstrate how the $p$-form fields appear upon the introduction of non-abelian gauge interactions. Section 3 first describes the pattern obtained for the $p$-form representations for maximal supergravity with space-time dimensions $d=3, \ldots, 7$. In a separate Sect. 3.1 we then try to generalize this pattern and show that it is in fact more generic. In a second Sect. 3.2 we describe how the representation content of the $p$-form gauge fields can be connected to results obtained in M-theory in a completely different context. In Sect. 4 we deal with generic gauge theories in four space-time dimensions in order to explain a number of features relevant for the $p$-form hierarchy in some more detail.

\section{Kaluza-Klein theory and gauge deformations}

Supergravity is an extension of general relativity that, in addition, is invariant under local supersymmetry, which transforms fermionic into bosonic fields and vice versa. Assuming that supergravity is an interacting field theory based on a finite number of fields, and that it allows a flat Minkowski space-time with maximal supersymmetry as a solution, it can be realized in at most $D=11$ space-time dimensions with the number of independent supersymmetries restricted to 32. Supergravity in eleven space-time dimensions [7] involves only three fields, namely a graviton field $g_{\mu \nu}$, a 3-form gauge field $A_{\mu \nu \rho}$ and a gravitino field $\psi_{\mu}$. Space-time indices are consistently denoted by $\mu, v, \ldots$ and, in this case, take the values $\mu, v, \ldots=0,1,2, \ldots, 10$. In eleven spacetime dimensions spinors carry 32 components, so that $\psi_{\mu}$ is a 32-component vector spinor. Maximal supergravity theories in $d$ space-time dimensions can be obtained by compactifying $11-d$ dimensions on a hyper-torus $T^{11-d}$. These compactified theories exhibit a remarkable invariance group of so-called 'hidden symmetries'. Deformations of the toroidally compactified theories are generally possible by introducing gauge interactions whose corresponding gauge group is embedded into the hidden symmetry group. This will be the topic of the second Sect. 2.2. In a few specific 
cases alternative deformations are possible as well. Of course, compactifications on non-flat manifolds can also be considered, and most of them will involve a breaking of supersymmetry. Some of these compactifications may arise as the result of a gauge deformation of a toroidally compactified theory, but this possibility will be ignored below.

In the first Sect. 2.1 we discuss the emergence of the 'hidden symmetry' group, first in gravity possibly extended with a tensor and a scalar field, and subsequently in supergravity.

\subsection{Hidden symmetries}

As an introduction we first discuss toroidal compactifications of general relativity, or an extension thereof, following the approach of Kaluza and Klein, who originally started from five space-time dimensions [8,9]. We first discuss the so-called 'hidden symmetries', demonstrating how the corresponding symmetry group takes a more interesting form upon including additional fields in the higher-dimensional theory. Subsequently we restrict ourselves to the massless sector and study general deformations of these theories by introducing additional gauge interactions. Hence, consider general relativity in $D$ space-time dimensions, with $n$ dimensions compactified on the torus $T^{n}$, so that space-time decomposes according to

$$
\mathcal{M}_{D} \rightarrow \mathcal{M}_{d} \times T^{n}
$$

where $d=D-n$. The resulting $d$-dimensional theory then describes massless graviton states, $n$ abelian gauge fields (called Kaluza-Klein photons) and $\frac{1}{2} n(n+1)$ massless scalar fields, as well as an infinite tower of massive graviton states. Besides the $d$-dimensional general coordinate transformations and the abelian gauge transformations, the theory turns out to be invariant under the group $\operatorname{GL}(n)$, which is non-linearly realized on the massless scalar fields. The latter fields parameterize the $\mathrm{GL}(n) / \mathrm{SO}(n)$ maximally symmetric space. The massive fields are all charged and couple to the $n$ abelian gauge fields with quantized charges. This restricts the $\mathrm{GL}(n)$ invariance to a discrete subgroup $\operatorname{GL}(n, \mathbb{Z})$ which leaves the lattice of Kaluza-Klein charges invariant.

The pattern of dimensional compactification changes when the dimension $d$ of the lower-dimensional space-time becomes equal to three. In three space-time dimensions, gravitons no longer carry local degrees of freedom (only topological ones) and the degrees of freedom residing in the Kaluza-Klein photons can be carried by scalar fields (here and henceforth we suppress the massive fields to which these photons couple, and concentrate on the massless sector). Hence the massless sector of the theory can be entirely formulated in terms of $\frac{1}{2} n(n+3)$ scalar fields. The symmetry group is now extended from GL $(n)$ to $\operatorname{SL}(n+1)$ (which are of equal rank) and the scalar fields parameterize the space $\operatorname{SL}(n+1) / \mathrm{SO}(n+1)$, which reflects the extended symmetry.

The emergence of hidden symmetries is a well known phenomenon in dimensional compactification. The rank of the symmetry group in $d$ dimensions is always increased by $n$ as compared to the rank of the symmetry group in the original $D$-dimensional 
theory, where $n=D-d$ denotes the number of toroidally compactified dimensions. Part of these hidden symmetries can be derived directly from a subset of the gauge transformations in the higher-dimensional ancestor theory, but others are somewhat less obvious. In toroidal compactifications it can also be shown that, when the massless scalars parameterize a homogeneous space in higher dimensions, this will also be the case in lower dimensions.

The presence of tensor gauge fields in the $D$-dimensional theory introduces further structure. To demonstrate this, consider, for instance, the Lagrangian of general relativity coupled to an anti-symmetric 2-form tensor field $B_{\mu \nu}$ in $D$ space-time dimensions,

$$
\mathcal{L}_{D}=-\frac{1}{2} \sqrt{g} R-\frac{3}{4} \sqrt{g}\left(\partial_{[\mu} B_{\nu \rho]}\right)^{2} .
$$

Its toroidal compactification leads to the symmetry group $\operatorname{SO}(n, n ; \mathbb{Z})$, which again has rank $n$. The lower-dimensional theory describes massless states belonging to the graviton, the antisymmetric tensor, and $2 n$ spin- 1 and $n^{2}$ spin- 0 states. The massless scalars parameterize the space $\mathrm{SO}(n, n ; \mathbb{R}) /[\mathrm{SO}(n ; \mathbb{R}) \times \mathrm{SO}(n ; \mathbb{R})]$. Furthermore there will be a tower of massive graviton and antisymmetric tensor states. This generic pattern will now change in space-time dimensions $d \leq 5$. In $d=5$ dimensions an antisymmetric tensor gauge field can be dualized to a vector gauge field, whereas, in $d=4$ dimensions, a tensor gauge field can be converted into a scalar field.

To make the theory a bit more interesting, let us also include a scalar field in $D$ dimensions, which couples such that the theory is invariant under certain scale transformations. This means that the theory in $D$ dimensions has an invariance group of unit rank. Depending on how precisely this scalar field interacts, the following result may arise in $d$ dimensions (always assuming $n=D-d$ ),

$$
\begin{aligned}
& d>5: \mathrm{G}=\mathbb{R}^{+} \times \mathrm{SO}(n, n ; \mathbb{Z}) \quad(n, n) \text { vectors } \\
& d=5: \mathrm{G}=\mathbb{R}^{+} \times \mathrm{SO}(n, n ; \mathbb{Z}) \quad(n, n)+1 \text { vectors } \\
& d=4: \mathrm{G}=\mathrm{SL}(2 ; \mathbb{Z}) \times \mathrm{SO}(n, n ; \mathbb{Z}) \quad(n, n)+1 \text { vectors } \\
& d=3: \mathrm{G}=\mathrm{SO}(n+1, n+1 ; \mathbb{Z}) \quad \text { no vectors }
\end{aligned}
$$

All these symmetry groups have rank $n+1$, in agreement with the general theorem. The massless scalars always parameterize a homogeneous space, namely $\operatorname{SO}(n, n ; \mathbb{R})$ / $[\mathrm{SO}(n ; \mathbb{R}) \times \mathrm{SO}(n ; \mathbb{R})]$, which, for $d=4$, is multiplied by a $\operatorname{SL}(2 ; \mathbb{R}) / \operatorname{SO}(2 ; \mathbb{R})$ factor.

As is well known, supergravity leads to a large variety of such hidden symmetry groups, including some of the exceptional groups. This is shown in Table 1, where we list the symmetry group $\mathrm{G}$ and its maximal compact subgroup $\mathrm{H}$ for maximal supergravities in space-time dimensions $d=3, \ldots, 7$. In supergravity the symmetry group is usually called the duality group, and in this context we will consistently use this nomenclature. The massless scalar fields then parameterize the homogeneous space $\mathrm{G} / \mathrm{H}$, and $\mathrm{H}$ coincides with the so-called R-symmetry group. The latter is the subgroup of the automorphism group of the supersymmetry algebra that commutes with the $d$-dimensional Lorentz transformations.

What we will be interested in is to study all possible deformations of supergravity theories that are induced by non-abelian gauge interactions. The corresponding gauge group must obviously be a subgroup of the duality group. The gauge fields, which so 
Table 1 Decomposition of the embedding tensor $\Theta$ for maximal supergravities in various space-time dimensions in terms of irreducible $\mathrm{G}$ representations

\begin{tabular}{llll}
\hline$d$ & $\mathrm{G}$ & $\mathrm{H}$ & $\Theta$ \\
\hline 7 & $\mathrm{SL}(5)$ & $\mathrm{USp}(4)$ & $10 \times 24=10+\underline{15}+\underline{40}+175$ \\
6 & $\mathrm{SO}(5,5)$ & $\mathrm{USp}(4) \times \mathrm{USp}(4)$ & $16 \times 45=16+\underline{144}+560$ \\
5 & $\mathrm{E}_{6(6)}$ & $\mathrm{USp}(8)$ & $27 \times 78=27+\underline{351}+1728$ \\
4 & $\mathrm{E}_{7(7)}$ & $\mathrm{SU}(8)$ & $56 \times 133=56+\underline{912}+6480$ \\
3 & $\mathrm{E}_{8(8)}$ & $\mathrm{SO}(16)$ & $248 \times 248=\underline{1}+248+\underline{3875}+27000+30380$ \\
\hline
\end{tabular}

Only the underlined representations are allowed according to the representation constraint. The R-symmetry group $\mathrm{H}$ is the maximal compact subgroup of $\mathrm{G}$

far were abelian and coupled only to the massive modes, will now couple also to the massless fields. Henceforth the massive modes will be discarded. For lower dimensions one obviously faces a dilemma, because by dualizing the higher-rank form fields one increases the degree of symmetry. On the other hand one also decreases the number of gauge fields in this way, and thus seems to constrain the possible gauge groups. The most extreme example of this is encountered in three space-time dimensions, where all the gauge fields can in fact be dualized to scalars, upon which the hidden symmetry group is extended, but on the other hand, there seem to be no gauge fields left to induce the gauging.

This last puzzle was resolved in [4,5], for maximal supergravity in three space-time dimensions. This theory does not contain any vector fields, and has the symmetry group $\mathrm{E}_{8(8)}(\mathbb{R}) .{ }^{1}$ Namely one adds a Chern-Simons term for as many gauge fields as there are rigid (continuous) invariances. For maximal supergravity in three dimensions this implies that one introduces 248 gauge fields, associated with the hidden symmetry group $\mathrm{E}_{8(8)}(\mathbb{R})$,

$$
\mathcal{L}_{\mathrm{CS}} \propto g \varepsilon^{\mu \nu \rho} A_{\mu}{ }^{M} \Theta_{M N}\left[\partial_{\nu} A_{\rho}{ }^{N}-\frac{1}{3} g f_{P} Q^{N} A_{\nu}{ }^{P} A_{\rho} Q\right]
$$

where $g$ is the gauge coupling constant, $f_{P} Q^{N}$ denotes the structure constants associated with a subgroup of $\mathrm{E}_{8(8)}(\mathbb{R})$, and $M, N, \ldots=1,2, \ldots, 248$. Furthermore, $\Theta_{M N}$ is a numerical symmetric tensor named the embedding tensor, which encodes the embedding of the gauge group in $\mathrm{E}_{8(8)}(\mathbb{R})$. The structure constants $f_{M N}{ }^{P}$ are implicitly encoded in the embedding tensor, in a way that we will exhibit below. In the next subsection we will introduce the embedding tensor in a more general context, where it is not necessarily a symmetric tensor. As we shall see, this set-up leads naturally to the introduction of a hierarchy of $p$-form fields.

\footnotetext{
1 A similar approach was followed subsequently for non-maximal supergravity in three space-time dimensions [10] (see also [11]).
} 


\subsection{Gauge deformations}

We start with a theory with abelian gauge fields $A_{\mu}{ }^{M}$, that is invariant under a group $\mathrm{G}$ of rigid transformations. The gauge fields transform in a representation $\mathcal{R}_{\mathrm{v}}$ of that group. ${ }^{2}$ The generators in this representation are denoted by $\left(t_{\alpha}\right)_{M}{ }^{N}$, so that $\delta A_{\mu}{ }^{M}=-\Lambda^{\alpha}\left(t_{\alpha}\right)_{N}{ }^{M} A_{\mu}{ }^{N}$, and the structure constants $f_{\alpha \beta}{ }^{\gamma}$ of $\mathrm{G}$ are defined according to $\left[t_{\alpha}, t_{\beta}\right]=f_{\alpha \beta}{ }^{\gamma} t_{\gamma}$. The next step is to select a subgroup of $\mathrm{G}$ that will be elevated to a gauge group with non-trivial gauge charges, whose dimension is obviously restricted by the number of vector fields. The discussion in this section will remain rather general and will neither depend on $\mathrm{G}$ nor on the space-time dimension. We refer to $[1,4,12-14]$ where a number of results was described for maximal supergravity in various dimensions.

The gauge group embedding is defined by specifying its generators $X_{M},{ }^{3}$ which couple to the gauge fields $A_{\mu}{ }^{M}$ in the usual fashion, and which can be decomposed in terms of the independent G-generators $t_{\alpha}$, i.e.,

$$
X_{M}=\Theta_{M}{ }^{\alpha} t_{\alpha}
$$

where $\Theta_{M}^{\alpha}$ is the embedding tensor transforming according to the product of the representation conjugate to $\mathcal{R}_{\mathrm{v}}$, the representation in which the gauge fields transform, and the adjoint representation of $\mathrm{G}$. This product representation is reducible and decomposes into a number of irreducible representations. Only a subset of these representations is allowed. For supergravity the precise constraints on the embedding tensor follow from supersymmetry, but from all applications worked out so far, we know that at least part (if not all) of the representation constraints is also required for purely bosonic reasons, such as gauge invariance of the action and consistency of the tensor gauge algebra. This constraint on the embedding tensor is known as the representation constraint. In Table 1 we have also included the representation constraints for maximal supergravity with $d=3, \ldots, 7$. It is important to note that we will always treat the embedding tensor as a spurionic object, which we allow to transform under $\mathrm{G}$, so that the Lagrangian and transformation rules remain formally G-invariant. Only at the end we will freeze the embedding tensor to a constant, so that the G-invariance will be broken. As was shown in $[6,15]$ this last step can also be described in terms of a new action in which the freezing of $\Theta_{M}{ }^{\alpha}$ will be the result of a more dynamical process. This will be discussed in due course.

The embedding tensor must satisfy a second constraint, the so-called closure constraint, which is quadratic in $\Theta_{M}{ }^{\alpha}$ and more generic. This constraint ensures that the gauge transformations form a group so that the generators (2.5) will close under commutation. Any embedding tensor that satisfies the closure constraint, together with the representation constraint mentioned earlier, defines a consistent gauging. The closure

\footnotetext{
2 In even space-time dimensions this assignment may fail and complete $\mathrm{G}$ representations may require the presence of magnetic duals. This was first demonstrated in [3] in four space-time dimensions.

3 The corresponding gauge algebra may have a central extension acting exclusively on the vector fields.
} 
constraint reads as follows,

$$
\mathcal{Q}_{P M}{ }^{\alpha}=\Theta_{P}{ }^{\beta} t_{\beta M}{ }^{N} \Theta_{N}{ }^{\alpha}+\Theta_{P}{ }^{\beta} f_{\beta \gamma}{ }^{\alpha} \Theta_{M}{ }^{\gamma} \approx 0
$$

and can be interpreted as the condition that the embedding tensor should be invariant under the embedded gauge group. Hence we can write the closure constraint as,

$$
\mathcal{Q}_{M N}{ }^{\alpha} \equiv \delta_{M} \Theta_{N}^{\alpha}=\Theta_{M}^{\beta} \delta_{\beta} \Theta_{N}^{\alpha} \approx 0,
$$

where $\delta_{M}$ and $\delta_{\alpha}$ denote the effect of an infinitesimal gauge transformation or an infinitesimal G-transformation, respectively. We indicate that $\mathcal{Q}_{M N}{ }^{\alpha}$ is "weakly zero" $\left(\mathcal{Q}_{M N}{ }^{\alpha} \approx 0\right)$ because later on we will introduce a description where the closure constraint will be imposed by certain field equations. Contracting (2.6) with $t_{\alpha}$ leads to,

$$
\left[X_{M}, X_{N}\right] \approx-X_{M N}^{P} X_{P}
$$

It is noteworthy here that the generator $X_{M N}{ }^{P}$ and the structure constants of the gauge group are related, but do not have to be identical. In particular $X_{M N}{ }^{P}$ is in general not antisymmetric in $[M N]$. The embedding tensor acts as a projector, and only in the projected subspace the matrix $X_{M N}{ }^{P}$ is antisymmetric in $[M N]$ and the Jacobi identity will be satisfied. Therefore (2.8) implies in particular that $X_{(M N)} P$ must vanish when contracted with the embedding tensor. Denoting

$$
Z_{M N}^{P} \equiv X_{(M N)}^{P},
$$

this condition reads,

$$
\Theta_{P}^{\alpha} Z^{P}{ }_{M N}=\mathcal{Q}_{(M N)}{ }^{\alpha} \approx 0 \text {. }
$$

The tensor $Z^{P}{ }_{M N}$ is constructed by contraction of the embedding tensor with G-invariant tensors and therefore transforms in the same representation as $\Theta_{M}{ }^{\alpha}-$ except when the embedding tensor transforms reducibly so that $Z^{P}{ }_{M N}$ may actually depend on a smaller representation. The closure constraint (2.7) ensures that $Z^{P}{ }_{M N}$ is gauge invariant. As is to be expected, $Z^{P}{ }_{M N}$ characterizes the lack of closure of the generators $X_{M}$. This can be seen, for instance, by calculating the direct analogue of the Jacobi identity (in the remainder of this section we assume that the closure constraint is identically satisfied),

$$
X_{[N P}^{R} X_{Q] R}{ }^{M}=\frac{2}{3} Z_{R[N}^{M} X_{P Q]}^{R} .
$$

The fact that the right-hand side does not vanish has direct implications for the nonabelian field strengths: the standard expression

$$
\mathcal{F}_{\mu \nu}{ }^{M}=\partial_{\mu} A_{\nu}{ }^{M}-\partial_{\nu} A_{\mu}{ }^{M}+g X_{N P}{ }^{M} A_{[\mu}{ }^{N} A_{\nu]}{ }^{P},
$$


which appears in the commutator $\left[D_{\mu}, D_{\nu}\right]=-g \mathcal{F}_{\mu \nu}{ }^{M} X_{M}$ of covariant derivatives

$$
D_{\mu} \equiv \partial_{\mu}-g A_{\mu}{ }^{M} X_{M}
$$

is not fully covariant. Rather, under standard gauge transformations

$$
\delta A_{\mu}{ }^{M}=D_{\mu} \Lambda^{M}=\partial_{\mu} \Lambda^{M}+g A_{\mu}{ }^{N} X_{N P}{ }^{M} \Lambda^{P},
$$

the field strength $\mathcal{F}_{\mu \nu}{ }^{M}$ transforms as

$$
\begin{aligned}
\delta \mathcal{F}_{\mu \nu}{ }^{M} & =2 D_{[\mu} \delta A_{\nu]}{ }^{M}-2 g Z^{M}{ }_{P Q} A_{[\mu}^{P} \delta A_{\nu]}{ }^{Q} \\
& =g \Lambda^{P} X_{N P}{ }^{M} \mathcal{F}_{\mu \nu}{ }^{N}-2 g Z^{M}{ }_{P Q} A_{[\mu}{ }^{P} \delta A_{\nu]} Q .
\end{aligned}
$$

This expression is not covariant, not only because of the presence of the second term on the right-hand side, but also because the lack of antisymmetry of $X_{N P}{ }^{M}$ prevents us from obtaining the expected result by inverting the order of indices $N P$ in the first term on the right-hand side. As a consequence, we cannot use $\mathcal{F}_{\mu \nu}{ }^{M}$ in the Lagrangian, because one needs suitable covariant field strengths for the invariant kinetic term of the gauge fields.

To remedy this lack of covariance, the strategy followed in $[1,2]$ has been to introduce additional (shift) gauge transformations on the vector fields,

$$
\delta A_{\mu}{ }^{M}=D_{\mu} \Lambda^{M}-g Z_{N P}^{M} \Xi_{\mu}^{N P},
$$

where the transformations proportional to $\Xi_{\mu}{ }^{N P}$ enable one to gauge away those vector fields that are in the sector of the gauge generators $X_{M N}{ }^{P}$ in which the Jacobi identity is not satisfied (this sector is perpendicular to the embedding tensor by (2.10)). Fully covariant field strengths can then be defined upon introducing 2-form tensor fields $B_{\mu \nu}{ }^{N P}$ belonging to the same representation as $\Xi_{\mu}{ }^{N P}$,

$$
\mathcal{H}_{\mu \nu}{ }^{M}=\mathcal{F}_{\mu \nu}{ }^{M}+g Z^{M}{ }_{N P} B_{\mu \nu}{ }^{N P} .
$$

These tensors transform covariantly under gauge transformations

$$
\delta \mathcal{H}_{\mu \nu}{ }^{M}=-g \Lambda^{P} X_{P N}{ }^{M} \mathcal{H}_{\mu \nu}{ }^{N}
$$

provided we impose the following transformation law for the 2-forms

$$
Z^{M P} \delta B_{\mu \nu}{ }^{N P}=Z^{M}{ }_{N P}\left(2 D_{[\mu} \Xi_{\nu]}{ }^{N P}-2 \Lambda^{N} \mathcal{H}_{\mu \nu}{ }^{P}+2 A_{[\mu}{ }^{N} \delta A_{\nu]}{ }^{P}\right) .
$$

We note that the constraint (2.10) ensures that

$$
\left[D_{\mu}, D_{\nu}\right]=-g \mathcal{F}_{\mu \nu}{ }^{M} X_{M}=-g \mathcal{H}_{\mu \nu}{ }^{M} X_{M}
$$

but in the Lagrangian the difference between $\mathcal{F}^{M}$ and $\mathcal{H}^{M}$ is important. 
Consistency of the gauge algebra thus requires the introduction of 2-form tensor fields $B_{\mu \nu}{ }^{P N}$. It is important that their appearance in (2.17) strongly restricts their possible representation content. Not only must they transform in the symmetric product $(N P)$ of the vector field representation as is manifest from their index structure, but also they appear under contraction with the tensor $Z^{M}{ }_{N P}$ which in general does not map onto the full symmetric tensor product in its lower indices, but rather only on a restricted sub-representation. We will see this explicitly in Sect. 4. It is this sub-representation of $\mathrm{G}$ to which the 2-forms are assigned, and to keep the notation transparent, we denote the corresponding projector with special brackets $[N P\rfloor$, such that

$$
Z_{N P}^{M} B_{\mu \nu}^{N P}=Z_{N P}^{M} B_{\mu \nu}^{\lceil N P\rfloor} \text {, etc. }
$$

The tensor $Z^{M}{ }_{N P}$ thus plays the role of an intertwiner between vector fields and 2 -forms, which encodes the precise field content of the 2-form tensor fields such that the consistency of the vector gauge algebra is ensured.

The same pattern continues upon definition of a covariant field strength for the 2 -forms and leads to a hierarchy of $p$-form tensor fields, which is entirely determined by the choice of the global symmetry group $\mathrm{G}$ and its fundamental representation $\mathcal{R}_{\mathrm{V}}$ in which the vector fields transform. In principle this hierarchy continues indefinitely, but it depends on the actual Lagrangian what its fate will be. Obviously, $p$ can at most be equal to $d$. When incorporated into a given Lagrangian the gauge algebra for the $p$-forms will be deformed and additional structure will appear. Some of the $p$-form gauge fields may carry physical degrees of freedom so they must already be contained in the ungauged Lagrangian, up to tensor dualities. For instance, in five dimensions, a vector gauge field and a 2 -form gauge field are dual, so that tensor fields are potentially present in view of the fact that the ungauged Lagrangian contains vector fields (which are also essential for the gauging). This is a generalization of the phenomenon we noted before: in three dimensions a scalar and a vector field can be dual. Therefore, vector fields are in principle available as well, as long as the ungauged Lagrangian contains scalar fields. The construction based on the Chern-Simons term (c.f. (2.4)) made use of this observation.

Before discussing these topics any further, let us first turn to a discussion of the hierarchy for the maximal supergravities. In Sect. 4 we will try to further elucidate the structure of the hierarchy for the case of a generic gauge theory in four space-time dimensions.

\section{The $p$-form hierarchy for the maximal supergravities}

The hierarchy of vector and tensor gauge fields that we discussed in the previous section can be considered for the maximal gauged supergravities. In that case the gauge group is embedded in the duality group $\mathrm{G}$, which is known for each space-time dimension in which the supergravity is defined (see Table 1). Once the group $G$ is specified, the hierarchy allows a unique determination of the representations of the higher $p$-forms. Table 2 illustrates this for the maximal supergravities in $d=3, \ldots, 7$ 
Table 2 Duality representations of the vector and tensor gauge fields for gauged maximal supergravities in space-time dimensions $3 \leq d \leq 7$

\begin{tabular}{llllllll}
\hline & & 1 & 2 & 3 & 4 & 5 & 6 \\
\hline 7 & $\mathrm{SL}(5)$ & $\overline{10}$ & 5 & $\overline{5}$ & 10 & 24 & $\overline{15}+40$ \\
6 & $\mathrm{SO}(5,5)$ & $16_{c}$ & 10 & $16_{s}$ & 45 & $144_{s}$ & $10+126_{s}+320$ \\
5 & $\mathrm{E}_{6(6)}$ & $\overline{27}$ & 27 & 78 & 351 & $27+1728$ & \\
4 & $\mathrm{E}_{7(7)}$ & 56 & 133 & 912 & $133+8645$ & & \\
3 & $\mathrm{E}_{8(8)}$ & 248 & $1+3875$ & $3875+147250$ & & & \\
\hline
\end{tabular}

The first two columns list the space-time dimension and the corresponding duality group

space-time dimensions. We recall that the analysis described in Sect. 2.2 did not depend on the number of space-time dimensions. For instance, it is possible to derive the representation assignments for $(d+1)$-rank tensors, although these do not live in a $d$-dimensional space-time (nevertheless, a glimpse of their existence occurs in $d$ dimensions via the shift transformations of the $d$-forms in the general gauged theory, as we shall see in due course). We also observe that the Hodge duality between the $p$-form fields that relates the $p$-forms to the $(d-p-2)$-forms is reflected in Table 2 , as the dual form fields appear in conjugate representations of the group $\mathrm{G}$. This duality depends, of course, sensitively on the space-time dimension, whereas the only input in the table came from the duality group and the representations of the low- $p$ form fields.

It is intriguing that the purely group theoretical hierarchy reproduces the correct assignments consistent with Hodge duality. In particular, the assignment of the $(d-2)$-forms is in line with tensor-scalar duality, as these forms are dual to the Noether currents associated with the G symmetry. In this sense, the duality group $\mathrm{G}$ implicitly carries information about the space-time dimension. But the hierarchy naturally extends beyond the $(d-2)$-forms and thus to those non-propagating forms whose field content is not subject to Hodge duality. It is another striking feature of the hierarchy that the diagonals pertaining to the $(d-1)$ - and $d$-rank tensor fields refer to the representations conjugate to those assigned to the embedding tensor and its quadratic constraint, respectively. This pattern is in fact generic and related to the special role these forms may play in the Lagrangian $[6,15]$. We will briefly discuss this in the next subsection in a general context. In a later Sect. 4 we will illustrate some of this in the context of a generic gauge theory in four space-time dimensions.

We recall that the embedding tensor is regarded as a so-called spurionic quantity, which transforms under the action of $\mathrm{G}$, although at the end it will be fixed to a constant value. For a specific value of the embedding tensor one is describing a given gauge deformation. Sweeping out the full space of allowed embedding tensors yields a $\Theta$-independent (and G-covariant) result for the representation of $p$-forms, as is shown in the table. This approach shows how the required consistency under generic gauge deformations imposes strong restrictions on the field content of the theory. In the ungauged theory there is a priori no direct evidence for these restrictions, but in certain 
cases there are alternative arguments, based, for instance, on supersymmetry or on underlying higher-rank Kac-Moody symmetries, which may motivate the representation content of the $p$-forms. Some of this will be discussed in Sect. 3.2.

\subsection{More about the hierarchy}

Now that we have seen some global features of the $p$-form algebra, let us briefly discuss some more generic aspects. As it turns out the $p$-forms transform in a sub-representation of the rigid symmetry group $\mathrm{G}$ of the theory that is contained in the $p$-fold tensor product $\mathcal{R}_{\mathrm{V}}^{\otimes p}$, where $\mathcal{R}_{\mathrm{V}}$ denotes the representation of $\mathrm{G}$ in which the vector fields transform. In many cases of interest this is the fundamental representation. We denote these fields by

$$
\stackrel{[1]}{A} M, \quad \stackrel{[2]}{B}[M N\rfloor, \quad \stackrel{[3]}{C}[M\lceil N P\rfloor\rfloor, \quad \stackrel{[4]}{C}[M\lceil N\lceil P Q\rfloor\rfloor], \quad \stackrel{[5]}{C}[M\lceil N\lceil P\lceil Q R] \cdot \cdot], \text { etc. },
$$

where we have suppressed space-time indices, and the special brackets $\lceil\cdots\rfloor$ are introduced to denote the relevant sub-representations of $\mathcal{R}_{\mathrm{V}}^{\otimes p}$, just as was done for $p=2$ in (2.21).

Assuming that the theory contains $p$-form fields and that one must also allow for the presence of the dual $p$-forms, the question arises what the significance is of the $d$ - and ( $d-1)$-forms that appear in Table 2, as those are not dual to any other forms. In order to explore this further, let us consider the high- $p$ sector of the hierarchy, starting with the $(d-3)$ - and the $(d-2)$-forms, transforming in the representations that are conjugate to those assigned to the 1- and the 2-forms [15]. In view of the fact that the theory is invariant under the group $G$ prior to switching on the gauge couplings, there exists a set of conserved 1-forms given by the Noether currents, transforming in the adjoint representation, which are dual to the $(d-2)$-forms. Hence we expect these forms to belong to the adjoint representation. Furthermore we expect $(d-3)$-forms that are dual to the vector fields, to transform in the G-representation $\mathcal{R}_{\mathrm{V} *}$, which is conjugate to the vector field representation $\mathcal{R}_{\mathrm{V}}$. When considering these high-rank $p$-forms it is convenient to switch to a notation adapted to this particular field content and to identify the $(d-3)$ - and $(d-2)$-forms as,

$$
\begin{aligned}
& \stackrel{[d-3]}{C} M_{1}\left[M_{2}\left[\cdots M_{d-3}\right] \cdots\right] \sim \stackrel{[d-3]}{C}_{M}, \\
& \stackrel{[d-2]}{C} M_{1}\left[M_{2}\left[\cdots M_{d-2}\right] \cdots\right] \sim \stackrel{[d-2]}{C}_{\alpha} .
\end{aligned}
$$

We may then explicitly study the end of the $p$-form hierarchy by imposing the general structure in a schematic form,

$$
\begin{aligned}
\delta \stackrel{[d-3]}{C}_{M} & =(d-3) \mathrm{D} \stackrel{[d-4]}{\Phi}_{M}+\cdots-g Y_{M} \stackrel{[d-3]}{\Phi}_{\alpha}, \\
\delta \stackrel{[d-2]}{C}_{\alpha} & =(d-2) \mathrm{D} \stackrel{[d-3]}{\Phi}_{\alpha}+\cdots-g Y_{\alpha, M}{\stackrel{[d-2]}{\Phi} M_{\beta},}^{[d-},
\end{aligned}
$$




$$
\begin{aligned}
& \delta \stackrel{[d-1]}{C}^{M}{ }_{\alpha}=(d-1) \mathrm{D} \stackrel{[d-2]}{\Phi} M_{\alpha}+\cdots-g Y_{\alpha, P Q}{ }^{\beta} \stackrel{[d-1]}{\Phi}^{M} Q_{\beta}, \\
& \delta \stackrel{[d]}{C}^{M N}{ }_{\alpha}=d \mathrm{D} \stackrel{[d-1]}{\Phi} M N_{\alpha}+\cdots-g Y_{\alpha, P Q R}{ }^{M N} \stackrel{[d]}{\Phi}^{P Q} Q_{\beta}, \\
& \delta \stackrel{[d+1]}{C}^{P} P R_{\alpha}=(d+1) \mathrm{D} \stackrel{[d]}{\Phi} P Q R_{\alpha}+\cdots,
\end{aligned}
$$

where we have indicated the most characteristic terms in the $p$-form transformations. We included the transformations associated to the $(d+1)$-form. Although this form does not exist in $d$ dimensions, its associated gauge transformations still play a role as they act on the $d$-form gauge fields, as is exhibited above.

The above schematic result (3.3), as well as the rest of the hierarchy, contains several new intertwining tensors $Y$ that connect the representations associated with two successive form fields. Formally they may be considered as a map

$$
Y^{[p]}: \mathcal{R}_{\mathrm{v}}^{\otimes(p+1)} \longrightarrow \mathcal{R}_{\mathrm{v}}^{\otimes p}
$$

which has a non-trivial kernel whose complement defines the representation content of the $(p+1)$-forms that is required for consistency of the deformed $p$-form gauge algebra. The lowest-rank intertwining tensors are given by

$$
Y^{[0]}: \mathcal{R}_{\mathrm{v}} \longrightarrow \mathcal{R}_{\mathrm{adj}}, \quad Y^{[1]}: \mathcal{R}_{\mathrm{v}}^{\otimes 2} \longrightarrow \mathcal{R}_{\mathrm{v}}
$$

corresponding to $p=0$, 1 , with $\left(Y^{[0]}\right)^{\alpha}{ }_{M}=\Theta_{M}{ }^{\alpha}$ and $\left(Y^{[1]}\right)^{M}{ }_{P Q}=Z^{M}{ }_{P Q}$. For higher $p$, the intertwining tensors can be defined recursively, as was demonstrated in [6]. All intertwining tensors are proportional to the embedding tensor and they must be mutually orthogonal,

$$
Y^{[p]} \cdot Y^{[p+1]} \approx 0
$$

This is a generalization of (2.10). More explicitly, the orthogonality relations read,

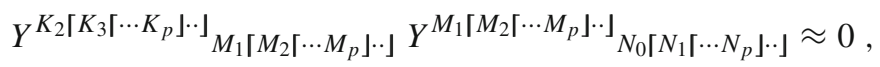

where 'weakly zero' indicates that the expression vanishes as a consequence of the quadratic constraint (2.6) on the embedding tensor.

It can be shown that the intertwining tensors appearing in (3.3) take the form, [15],

$$
\begin{aligned}
Y_{M}{ }^{\alpha}= & \Theta_{M}{ }^{\alpha}, \\
Y_{\alpha, M}{ }^{\beta}= & \delta_{\alpha} \Theta_{M}^{\beta}, \\
Y^{M}{ }_{\alpha, P Q}^{\beta}= & -\frac{\partial \mathcal{Q}_{P Q}{ }^{\beta}}{\partial \Theta_{M}^{\alpha}}, \\
Y^{M N}{ }_{\alpha, P Q R}{ }^{\beta}= & -\delta_{P}^{M} Y^{N}{ }_{\alpha, Q R}^{\beta}-X_{P Q}{ }^{M} \delta_{R}^{N} \delta_{\alpha}^{\beta} \\
& -X_{P R}{ }^{N} \delta_{Q}^{M} \delta_{\alpha}^{\beta}+X_{P \alpha}{ }^{\beta} \delta_{R}^{N} \delta_{Q}^{M} .
\end{aligned}
$$


It is straightforward to verify that these intertwining tensors satisfy the mutual orthogonality property (3.7), and one easily derives,

$$
\begin{aligned}
Y_{M}{ }^{\alpha} Y_{\alpha, N}{ }^{\beta} & \approx 0, \\
Y_{\alpha, N}{ }^{\beta} Y_{\beta, P Q}{ }^{\gamma} & \approx 0, \\
Y_{\alpha, K L}{ }^{\beta} Y_{\beta, P Q R}{ }^{\gamma}{ }_{\beta,} & \approx 0 .
\end{aligned}
$$

There is an additional identity which holds identically, without making reference to the constraint (2.7),

$$
Y^{M N}{ }_{\alpha, P Q R}^{\beta} \mathcal{Q}_{M N}{ }^{\alpha}=0
$$

The relevance of this result will be discussed below.

From (3.8) we can now directly read off the representation content of the $(d-1)$ and the $d$-forms that follows from the hierarchy: the form of $Y_{\alpha, M}{ }^{\beta}$ and $Y^{M}{ }_{\alpha, P} Q^{\beta}$ shows that these forms transform in the representations dual to the embedding tensor $\Theta_{M}{ }^{\beta}$ and the quadratic constraint $\mathcal{Q}_{P Q} Q^{\beta}$, respectively. As such, they can naturally be coupled, acting as Lagrange multipliers enforcing the property that the embedding tensor is space-time independent and gauge invariant [6]. This idea has been worked out explicitly in the context of maximal supergravity in three space-time dimensions, and subsequently it has been argued that this situation can also be realized in a more general context [15]. Hence we view the embedding tensor as a space-time dependent scalar field, transforming in the G-representation that is allowed by the representation constraint. To the original Lagrangian $\mathcal{L}_{0}$ which may depend on $p$-forms with $p \leq d-2$, we then add the following terms,

$$
\mathcal{L}=\mathcal{L}_{0}+\mathcal{L}_{\mathrm{C}}
$$

with

$$
\mathcal{L}_{\mathrm{C}} \propto \varepsilon^{\mu_{1} \cdots \mu_{d}}\left\{d g C_{\mu_{2} \cdots \mu_{d}}{ }^{M}{ }_{\alpha} D_{\mu_{1}} \Theta_{M}{ }^{\alpha}+g^{2} C_{\mu_{1} \cdots \mu_{d}}{ }^{M N}{ }_{\alpha} \mathcal{Q}_{M N}{ }^{\alpha}\right\} .
$$

Note that the identity (3.10) ensures that this Lagrangian is invariant under the shift transformation of the $d$-rank tensor field. Variation of this Lagrangian with respect to $\Theta_{M}^{\alpha}(x)$ leads to the following expression,

$$
\begin{aligned}
& \delta \mathcal{L}_{\mathrm{C}} \propto-g \varepsilon^{\mu_{1} \cdots \mu_{d}} \delta \Theta_{M}{ }^{\alpha} \\
& \quad \times\left[d D_{\mu_{1}} C_{\mu_{2} \cdots \mu_{d}}{ }^{M}{ }_{\alpha}+g Y^{M}{ }_{\alpha, P Q}{ }^{\beta} C_{\mu_{1} \cdots \mu_{d}}{ }^{P Q}{ }_{\beta}+d g A_{\mu_{1}} Y_{\alpha, N}{ }^{\beta} C_{\mu_{2} \cdots \mu_{d}}{ }^{N}{ }_{\beta}\right] .
\end{aligned}
$$

This result can be written as follows,

$$
\delta \mathcal{L}_{\mathrm{C}} \propto-g \varepsilon^{\mu_{1} \cdots \mu_{d}}\left[\mathcal{H}_{\mu_{1} \cdots \mu_{d}}{ }^{M}{ }_{\alpha}+d A_{\left[\mu_{1}\right.}{ }^{M} \mathcal{H}_{\left.\mu_{2} \cdots \mu_{d}\right] \alpha}+\cdots\right] \delta \Theta_{M}{ }^{\alpha},
$$


by including unspecified terms involving form fields of rank $p \leq d-2$. These terms are expected to arise from the $\Theta$-variation of the Lagrangian $\mathcal{L}_{0}$, but they cannot be evaluated in full generality as this depends on the details of the latter Lagrangian. We return to this issue in the next section where we study the situation in four space-time dimensions.

\subsection{M-theory}

It is an obvious question whether the systematic features shown in Table 2 have a natural explanation in terms of M-theory. Supergravity may already contain some of the fields that carry charges that are required for some of these gaugings. Indeed, we already noted in Sect. 2.1 that the towers of massive Kaluza-Klein states carry charges that couple to the Kaluza-Klein gauge fields emerging from the higher-dimensional metric. This is of direct relevance to the so-called Scherk-Schwarz reductions [16]. However, these Kaluza-Klein states cannot generally be assigned to representations of the duality group and therefore there must be extra degrees of freedom whose origin cannot be understood within the context of a dimensional compactification of supergravity. ${ }^{4}$ This phenomenon was discussed some time ago, for instance, in $[17,18]$.

General gaugings of maximal supergravity constructed in recent years obviously extend beyond gaugings whose charges can be fully understood from supergravity degrees of freedom in higher dimensions. The duality covariant embedding tensor encodes all the possible charges which must somehow have their origin in M-theory. Indeed there are indications that this is the case. In this way the gauging acts as a probe of M-theory degrees of freedom.

Independent evidence that this relation with M-theory degrees of freedom is realized, is provided by the work of [19] (see also, [17] and references quoted therein) where matrix theory $[20,21]$ is considered in a toroidal compactification. This work is based on the correspondence between $N=4$ super-Yang-Mills theory in $n+1$ dimensions $(n \leq 9)$, on a (rectangular) spatial torus $\tilde{T}^{n}$ with radii $s_{1}, \ldots, s_{n}$, and M-theory in the infinite-momentum frame on the dual torus $T^{n}$ with radii $R_{1}, R_{2}, \ldots, R_{n}$, where $s_{i}=l_{\mathrm{p}}^{3} / R_{11} R_{i}$. Here $l_{\mathrm{p}}$ denotes the Planck length in eleven dimensions and $R_{11}$ is the length of the compactified eleventh dimension. The latter dimension, together with the time dimension and the spatial dimensions that do not belong to $T^{n}$ constitute the $d$-dimensional space-time that is relevant in the comparison. Just as before $d=11-n$.

The conjecture is that M-theory should be invariant under both the permutations of the radii $R_{i}$ and under T-duality of type-IIA string theory. The relevant T-duality transformations follow from making two consecutive T-dualities on two different circles. When combined with the permutation symmetry, T-duality can be represented by $(i \neq j \neq k \neq i)$

$$
R_{i} \rightarrow \frac{l_{\mathrm{p}}^{3}}{R_{j} R_{k}}, \quad R_{j} \rightarrow \frac{l_{\mathrm{p}}^{3}}{R_{k} R_{i}}, \quad R_{k} \rightarrow \frac{l_{\mathrm{p}}^{3}}{R_{i} R_{j}}, \quad l_{\mathrm{p}}^{3} \rightarrow \frac{l_{\mathrm{p}}^{6}}{R_{i} R_{j} R_{k}}
$$

\footnotetext{
4 In view of the fact that the Kaluza-Klein states are 1/2-BPS, also these extra degrees of freedom must correspond to $1 / 2$-BPS states.
} 
The above transformations generate a discrete group which coincides with the Weyl group of $\mathrm{E}_{n}$; on the Yang-Mills side, the elementary Weyl reflections correspond to permutations of the compactified coordinates (generating the Weyl group of SL $(n)$ ) and to Montonen-Olive duality $g_{\text {eff }} \rightarrow 1 / g_{\text {eff }}$ (corresponding to reflections with respect to the exceptional node of the $\mathrm{E}_{n}$ Dynkin diagram). This Weyl group, which leaves the rectangular shape of the compactification torus invariant, can be realized as a discrete subgroup of the compact subgroup of $\mathrm{E}_{n(n)}$, and consequently as a subgroup of the conjectured non-perturbative duality group $\mathrm{E}_{n(n)}(\mathbb{Z})[22]$. Representations of this symmetry can now be generated by mapping out the Weyl orbits starting from certain states. For instance, one may start with Kaluza-Klein states on $T^{n}$, whose masses are proportional to $M \sim 1 / R_{i}$. The action of the Weyl group then generates new states, such as the ones that can be identified with two-branes wrapped around the torus, whose masses are of order $M \sim R_{j} R_{k} / l_{\mathrm{p}}^{3}$, and so on. To be specific, let us consider the situation for $n=4$ and $d=7$ and start from the four Kaluza-Klein states with masses $M \sim 1 / R_{i}$, where $i=1,2,3,4$. Upon the action of (3.15), we find six two-brane states wrapped on $T^{4}$. Repeated application of (3.15) does not give rise to new states, so that we find precisely ten particle states (i.e., massive charged particle states from the seven-dimensional perspective):

10 particle states $\begin{cases}4 \mathrm{KK} \text { states on } T^{4} & : M \sim \frac{1}{R_{i}} \\ 6 \text { two-brane states wrapped on } T^{4}: M \sim \frac{R_{j} R_{k}}{l_{\mathrm{p}}^{3}}\end{cases}$

Here $j \neq k$. The pointlike charges associated with these states can couple to ten gauge fields, and this is precisely the number of 1 -forms in Table 2 for $d=7$.

Likewise we can consider 2-brane states wrapped on $T^{4} \times \mathbb{R}^{11}$, where $\mathbb{R}^{11}$ denotes the eleventh dimension, which has been compactified to size $R_{11}$. There are four such states with masses $M \sim R_{11} R_{i} / l_{\mathrm{p}}^{3}$. Application of (3.15) now leads to only one additional state, corresponding to a five-brane wrapped on $T^{4} \times \mathbb{R}^{11}$. Hence we find five stringlike states, from the perspective of the seven-dimensional spacetime,

5 string states $\left\{\begin{array}{l}4 \text { two-brane states wrapped on } T^{4} \times \mathbb{R}^{11}: M \sim \frac{R_{11} R_{i}}{l_{\mathrm{p}}^{3}} \\ 1 \text { five-brane state wrapped on } T^{4} \times \mathbb{R}^{11}: M \sim \frac{R_{11} R_{1} R_{2} R_{3} R_{4}}{l_{\mathrm{p}}^{6}}\end{array}\right.$

Altogether we thus have a multiplet consisting of five different string states, which can couple to five different 2-form fields. This is precisely the number of 2-forms listed in Table 2 for $d=7$.

Similar arguments apply to the other states, except that when the representation has weights of different lengths, one needs several different Weyl orbits to recover all 
states in the representation. For instance, there are only 2160 states for $E_{8(8)}$, which must be supplemented by eight-brane states to obtain the full 3875 representation of $\mathrm{E}_{8(8)}$. In this way one obtains complete multiplets of the duality group (taking into account that some states belonging to the representation will vanish under the Weyl group and will therefore remain inaccessible by this construction).

The representations in the table were also found in [23], where a 'mysterious duality' was exhibited between toroidal compactifications of M-theory and del Pezzo surfaces. Here the M-theory dualities are related to global diffeomorphisms that preserve the canonical class of the del Pezzo surface. Again the representations thus found are in good agreement with the representations in Table 2.

For $n=9$, the multiplets given in [19] have infinitely many components. Indeed, there are hints that the above considerations concerning new M-theoretic degrees of freedom can be extended to infinite-dimensional duality groups. Already some time ago [24] it was shown from an analysis of the indefinite Kac-Moody algebra $\mathrm{E}_{11}$ that the decomposition of its so-called L1 representation at low levels under its finitedimensional subalgebra SL $(3) \times E_{8}$ yields the same 3875 representation that appears for the two-forms shown in Table 2 . This analysis has meanwhile been extended [2528] to other space-time dimensions and higher-rank forms, and again there is a clear overlap with the representations in Table 2. Nevertheless it remains far from clear what all these (infinitely many) new degrees of freedom would correspond to, and how they would be concretely realized. Concerning the physical interpretation of the new states, a first step was taken in [29], where an infinite multiplet of BPS states is generated from the M2 brane and M5 brane solutions of $D=11$ supergravity by the iterated action of certain $A_{1}^{(1)}$ subgroups of the $\mathrm{E}_{9}$ Weyl group. For more recent work, see [30]. In the context of gauged supergravities, the significance of these states may become clearer with the exploration of maximal gauged supergravities in two space-time dimensions [31], where the embedding tensor transforms in the so-called basic representation of $\mathrm{E}_{9}$ (which is infinite dimensional).

\section{The $p$-form hierarchy in four space-time dimensions}

In this section we present the $p$-form hierarchy for a generic $d=4$ dimensional gauge theory, following earlier work in [3,32-34]. Although matters will become more complicated towards the end, we hope that this illustrates a number of characteristic features. First of all, we will see that the $p$-form fields belong to restricted representations, as was noted previously. Then we will exhibit the fact that the gauge transformations of the hierarchy are deformed when considered in the context of a specific Lagrangian, rather than as an abstract algebra. And finally, we will be more explicit (although we will refrain from giving all the details) about the introduction of the 3- and 4-form fields. For simplicity we suppress the gravitational interactions and consider a Lagrangian depending on $n$ abelian gauge fields $A_{\mu}{ }^{\Lambda}$ (so that $\Lambda=1, \ldots, n$, where $n$ has no relation to the torus dimension, as in the previous sections). We start without charged fields so that the gauge fields $A_{\mu}{ }^{\Lambda}$ appear exclusively through the field strengths, $F_{\mu \nu}^{\Lambda}=2 \partial_{[\mu} A_{\nu]}{ }^{\Lambda}$. 
The field equations for these fields and the Bianchi identities for the field strengths comprise $2 n$ equations,

$$
\partial_{[\mu} F_{v \rho]}^{\Lambda}=0=\partial_{[\mu} G_{v \rho] \Lambda}
$$

where

$$
G_{\mu \nu \Lambda}=\varepsilon_{\mu \nu \rho \sigma} \frac{\partial \mathcal{L}}{\partial F_{\rho \sigma} \Lambda},
$$

where we use a metric with signature $(-,+,+,+)$ and $\varepsilon_{0123}=1$ denotes the fourdimensional Levi-Civita symbol in four Minkowskian dimensions.

It is convenient to combine the tensors $F_{\mu \nu} \Lambda$ and $G_{\mu \nu \Lambda}$ into a $2 n$-dimensional vector,

$$
G_{\mu \nu}{ }^{M}=\left(\begin{array}{l}
F_{\mu \nu}^{\Lambda} \\
G_{\mu \nu \Lambda}
\end{array}\right),
$$

so that (4.1) reads $\partial_{[\mu} G_{v \rho]}{ }^{M}=0$. Obviously, these $2 n$ equations are invariant under real $2 n$-dimensional rotations of the tensors $G_{\mu \nu}{ }^{M}$,

$$
\left(\begin{array}{l}
F^{\Lambda} \\
G_{\Lambda}
\end{array}\right) \longrightarrow\left(\begin{array}{l}
\tilde{F}^{\Lambda} \\
\tilde{G}_{\Lambda}
\end{array}\right)=\left(\begin{array}{ll}
U^{\Lambda} Z^{\Lambda \Sigma} \\
W_{\Lambda \Sigma} & V_{\Lambda}^{\Sigma}
\end{array}\right)\left(\begin{array}{l}
F^{\Sigma} \\
G_{\Sigma}
\end{array}\right)
$$

The first half of the rotated tensors can be adopted as new field strengths defined in terms of new gauge fields, and constraints on the remaining tensors can then be interpreted as field equations belonging to some new Lagrangian $\tilde{\mathcal{L}}$ expressed in terms of the new field strengths $\tilde{F}_{\mu \nu} \Lambda$, with $\tilde{G}_{\mu \nu}=\varepsilon_{\mu \nu \rho \sigma} \partial \tilde{\mathcal{L}} / \partial \tilde{F}_{\rho \sigma} \Lambda$. In order that such a Lagrangian exists, the real matrix in (4.4) must belong to the group $\operatorname{Sp}(2 n ; \mathbb{R})$. This group consists of real matrices that leave the skew-symmetric tensor $\Omega_{M N}$ invariant,

$$
\Omega=\left(\begin{array}{cc}
0 & 1 \\
-1 & 0
\end{array}\right)
$$

The conjugate matrix $\Omega^{M N}$ is defined by $\Omega^{M N} \Omega_{N P}=-\delta^{M}{ }_{P}{ }^{5}$ The $\operatorname{Sp}(2 n ; \mathbb{R})$ transformations are known as electric/magnetic dualities, which also act on electric and magnetic charges (for a review of electric/magnetic duality, see [35]). The Lagrangian depends on the electric/magnetic duality frame and is therefore not unique. Different Lagrangians related by electric/magnetic duality lead to equivalent field equations and Bianchi identities, and thus belong to the same equivalence class. Since the relationship (4.4) between the old and the new field strengths is not a local one, the new

5 Here we employ an $\operatorname{Sp}(2 n, \mathbb{R})$ covariant notation for the $2 n$-dimensional symplectic indices $M, N, \ldots$, such that $Z^{M}=\left(Z^{\Lambda}, Z_{\Lambda}\right)$. Likewise we use vectors with lower indices according to $Y_{M}=\left(Y_{\Lambda}, Y^{\Lambda}\right)$, transforming according to the conjugate representation so that $Z^{M} Y_{M}$ is invariant under $\operatorname{Sp}(2 n ; \mathbb{R})$. 
Lagrangian can in general not be obtained by straightforward substitution. Instead one may derive,

$$
\tilde{\mathcal{L}}(\tilde{F})+\frac{1}{8} \varepsilon^{\mu \nu \rho \sigma} \tilde{F}_{\mu \nu}{ }^{\Lambda} \tilde{G}_{\rho \sigma \Lambda}=\mathcal{L}(F)+\frac{1}{8} \varepsilon^{\mu \nu \rho \sigma} F_{\mu \nu}{ }^{\Lambda} G_{\rho \sigma \Lambda}
$$

up to terms independent of $F^{\mu \nu \Lambda}$. Clearly the Lagrangian does not transform as a function, since

$$
\tilde{\mathcal{L}}(\tilde{F}) \neq \mathcal{L}(F)
$$

but the combination,

$$
\mathcal{L}(F)+\frac{1}{8} \varepsilon^{\mu \nu \rho \sigma} F_{\mu \nu}{ }^{\Lambda} G_{\rho \sigma \Lambda},
$$

does, in view of (4.6). ${ }^{6}$

When $\mathcal{L}$ remains unchanged under the duality transformation, i.e. when

$$
\tilde{\mathcal{L}}(\tilde{F})=\mathcal{L}(\tilde{F})
$$

then the theory is invariant under the corresponding transformations. It is usually difficult to verify this equation explicitly. Instead one may verify that the substitution $F_{\mu \nu} \Lambda \rightarrow \tilde{F}_{\mu \nu}{ }^{\Lambda}$ into the derivatives $\partial \mathcal{L}(F) / \partial F^{\mu \nu} \Lambda$ correctly induces the symplectic transformations of the field strengths $G_{\mu \nu \Lambda}$. In this case, the linear combination (4.8), which can also be written as $\mathcal{L}(F)-\frac{1}{2} F_{\mu \nu} \Lambda \partial \mathcal{L}(F) / \partial F_{\mu \nu} \Lambda$, must be an invariant function under $F_{\mu \nu}{ }^{\Lambda} \rightarrow \tilde{F}_{\mu \nu}{ }^{\Lambda}$. Note that in the literature the word duality is used both for equivalence and for invariance transformations. But the duality group $\mathrm{G}$ introduced before, includes only those $\operatorname{Sp}(2 n ; \mathbb{R})$ transformations that satisfy (4.9).

For clarity we first consider a sub-class of the duality transformations consisting of those transformations (4.4) for which $Z=0$. These are the transformations that act locally on the various fields, as can be seen from the fact that $\tilde{F}_{\mu \nu}^{\Lambda}=U^{\Lambda}{ }_{\Sigma} F_{\mu \nu}{ }^{\Sigma}$. Because the transformation must belong to $\operatorname{Sp}(2 n ; \mathbb{R})$, it follows $U V^{\mathrm{T}}=\mathbf{1}$ and that $U^{\mathrm{T}} W$ is a symmetric matrix. Using (4.6) and (4.9), one easily derives that the Lagrangian changes by a total derivative,

$$
\mathcal{L}\left(U^{\Lambda}{ }_{\Sigma} F^{\Sigma}\right)=\mathcal{L}\left(F^{\Lambda}\right)-\frac{1}{8} \varepsilon^{\mu \nu \rho \sigma}\left(U^{\mathrm{T}} W\right)_{\Lambda \Sigma} F_{\mu \nu}{ }^{\Lambda} F_{\rho \sigma}{ }^{\Sigma}
$$

So far we have only indicated the dependence on the field strengths $F_{\mu \nu}{ }^{\Lambda}$, but other fields may be present as well and will transform locally among themselves. Their transformations have to be included in (4.9).

We now consider the introduction of a non-abelian gauge group that will act non-trivially on the vector fields and must therefore involve a subgroup of the duality group. Because the duality group acts both on electric and on magnetic charges, in

\footnotetext{
6 When the field equations of the vector fields are imposed, the Lagrangian does in fact transform as a function under electric/magnetic duality.
} 
view of the fact that it mixes field strengths with dual field strengths as shown by (4.4), we must eventually introduce magnetic gauge fields $A_{\mu \Lambda}$ as well, following the procedure explained in [3]. The $2 n$ gauge fields $A_{\mu}{ }^{M}$ will then comprise both type of fields, $A_{\mu}{ }^{M}=\left(A_{\mu}{ }^{\Lambda}, A_{\mu \Lambda}\right)$. The role played by the magnetic gauge fields will be clarified later. For the moment one may associate $A_{\mu \Lambda}$ with the dual field strengths $G_{\mu \nu \Lambda}$, by writing $G_{\mu \nu \Lambda} \equiv 2 \partial_{[\mu} A_{\nu] \Lambda}$.

The gauge group generators (as far as their embedding in the duality group is concerned) are then defined as follows. The generators of the subgroup that is gauged, are $2 n$-by- $2 n$ matrices $X_{M}$, where we are assuming the presence of both electric and magnetic gauge fields, so that the generators decompose according to $X_{M}=\left(X_{\Lambda}, X^{\Lambda}\right)$. Obviously $X_{\Lambda N}{ }^{P}$ and $X^{\Lambda}{ }_{N}{ }^{P}$ can be decomposed into the generators of the duality group and are thus consistent with the infinitesimal form of the transformations (4.4). Denoting the gauge group parameters by $\Lambda^{M}(x)=\left(\Lambda^{\Lambda}(x), \Lambda_{\Lambda}(x)\right), 2 n$-dimensional $\operatorname{Sp}(2 n ; \mathbb{R})$ vectors $Y^{M}$ and $Z_{M}$ transform according to

$$
\delta Y^{M}=-g \Lambda^{N} X_{N P}{ }^{M} Y^{P}, \quad \delta Z_{M}=g \Lambda^{N} X_{N M}{ }^{P} Z_{P},
$$

where $g$ denotes a universal gauge coupling constant. Covariant derivatives thus take the form,

$$
\begin{aligned}
D_{\mu} Y^{M} & =\partial_{\mu} Y^{M}+g A_{\mu}{ }^{N} X_{N P}{ }^{M} Y^{P} \\
& =\partial_{\mu} Y^{M}+g A_{\mu}{ }^{\Lambda} X_{\Lambda P}{ }^{M} Y^{P}+g A_{\mu \Lambda} X^{\Lambda}{ }_{P}{ }^{M} Y^{P}
\end{aligned}
$$

and similarly for $D_{\mu} Z_{M}$. The gauge fields then transform according to

$$
\delta A_{\mu}{ }^{M}=D_{\mu} \Lambda^{M}=\partial_{\mu} \Lambda^{M}+g X_{P Q}{ }^{M} A_{\mu}{ }^{P} \Lambda^{Q}
$$

After replacing ordinary by covariant derivatives and field strengths, the Lagrangian is in general not invariant. To see this, let us consider gauge transformations belonging to the subgroup considered earlier, for which the field transformations take a local form. Hence we set $X^{\Lambda}{ }_{N} P=0$ so that the magnetic gauge fields will not enter and $X_{\Lambda}{ }^{\Sigma \Gamma}=0$. In this case we can make use of the result (4.10). As before the Lagrangian is not invariant and it changes with the covariantized form of the variation (4.10),

$$
\mathcal{L} \rightarrow \mathcal{L}+\frac{1}{8} \varepsilon^{\mu \nu \rho \sigma} \Lambda^{\Lambda} X_{\Lambda \Sigma \Gamma} \mathcal{F}_{\mu \nu}{ }^{\Sigma} \mathcal{F}_{\rho \sigma}{ }^{\Gamma}
$$

where the tensors $\mathcal{F}_{\mu \nu}{ }^{\Lambda}$ denote the non-abelian field strengths,

$$
\mathcal{F}_{\mu \nu}{ }^{\Lambda}=\partial_{\mu} A_{\nu}{ }^{\Lambda}-\partial_{\nu} A_{\mu}{ }^{\Lambda}+g X_{\Sigma \Gamma}{ }^{\Lambda} A_{[\mu}{ }^{\Sigma} A_{\nu]}{ }^{\Gamma} .
$$

Consequently, the variation of the Lagrangian is no longer a total derivative when the gauge parameters are space-time dependent functions. To obtain a variation that is equal to a total derivative, one must include a new term in the Lagrangian [36],

$$
\mathcal{L}=\frac{1}{3} g \varepsilon^{\mu \nu \rho \sigma} X_{\Lambda \Sigma \Gamma} A_{\mu}{ }^{\Lambda} A_{\nu}{ }^{\Sigma}\left(\partial_{\rho} A_{\sigma}{ }^{\Gamma}+\frac{3}{8} g X_{\Xi \Delta}{ }^{\Gamma} A_{\rho}{ }^{\Xi} A_{\sigma}{ }^{\Delta}\right)
$$


In the case of more general gauge group embeddings, this term is not sufficient and extra terms will have to be introduced as soon as also the magnetic gauge fields are present.

We now consider more general gauge groups without restricting ourselves to electric charges. Therefore we must include both electric gauge fields $A_{\mu}{ }^{\Lambda}$ and magnetic gauge fields $A_{\mu \Lambda}$. Only a subset of these fields is usually involved in the gauging, but the additional magnetic gauge fields could conceivably lead to new propagating degrees of freedom. We will see in due course how this is avoided. The charges $X_{M N} P$ correspond to a more general subgroup of the duality group. Hence they must take values in the Lie algebra associated with $\operatorname{Sp}(2 n, \mathbb{R})$, which implies,

$$
X_{M[N}{ }^{Q} \Omega_{P] Q}=0 .
$$

Furthermore we impose the representation constraint that was discussed earlier. In this case the constraint implies that we suppress a representation of the rigid symmetry group in $X_{M N}{ }^{P}[3]$,

$$
X_{(M N} Q \Omega_{P) Q}=0 \Longrightarrow\left\{\begin{array}{l}
X^{(\Lambda \Sigma \Gamma)}=0, \\
2 X^{(\Gamma \Lambda)}=X_{\Sigma} \Lambda \Gamma \\
X_{(\Lambda \Sigma \Gamma)}=0, \\
X_{(\Gamma \Lambda)}{ }^{\Lambda}=X^{\Sigma}{ }_{\Lambda \Gamma} .
\end{array}\right.
$$

Observe that the generators $X_{\Lambda \Sigma} \Sigma^{\Gamma}$ are not necessarily antisymmetric in $\Lambda$ and $\Sigma$; their antisymmetric part appears in the field strengths (4.15). The symmetric part defines the tensor $Z^{P}{ }_{M N}$ according to (2.9). In the case at hand we derive, using (4.18),

$$
Z_{M N}^{P}=\frac{1}{2} \Omega^{P R} \Theta_{R}{ }^{\alpha} t_{\alpha M}{ }^{Q} \Omega_{N Q}
$$

which shows that the symmetric index pair $(M N)$ of $Z^{P}{ }_{M N}$ is restricted to the adjoint representation of the rigid symmetry group $\mathrm{G}$. Henceforth we use the notation,

$$
X_{(M N)}^{P}=Z_{M N}^{P}=Z^{P, \alpha} d_{\alpha M N},
$$

where

$$
\begin{aligned}
& d_{\alpha M N} \equiv\left(t_{\alpha}\right)_{M}{ }^{P} \Omega_{N P}, \\
& Z^{M, \alpha} \equiv \frac{1}{2} \Omega^{M N} \Theta_{N}{ }^{\alpha} \Longrightarrow\left\{\begin{array}{l}
Z^{\Lambda \alpha}=\frac{1}{2} \Theta^{\Lambda \alpha}, \\
Z_{\Lambda}{ }^{\alpha}=-\frac{1}{2} \Theta_{\Lambda}{ }^{\alpha} .
\end{array}\right.
\end{aligned}
$$

Note, however, that when the symmetry group $\mathrm{G}$ is not simple, then the indices $\alpha, \beta, \ldots$ above will be restricted to the invariant subgroup that acts on the vector fields. The tensor $Z^{M, \alpha}$ takes only non-zero values for those indices $\alpha$. Hence the 2-forms transform in a restricted sub-representation (namely the adjoint representation) of the symmetric tensor product, as we stressed earlier. 
Let us now consider the closure constraint (2.7), which gave rise to the orthogonality relation between the embedding tensor and the tensor $Z$. In the case at hand this implies,

$$
Z^{M \alpha} \Theta_{M}^{\beta} d_{\alpha P Q}=\frac{1}{2} \Omega^{M N} \Theta_{M}{ }^{\beta} \Theta_{N}{ }^{\alpha} d_{\alpha P Q}=\mathcal{Q}_{(P Q)}{ }^{\beta} \approx 0
$$

In case the gauge group is embedded into a simple group then $\Omega^{M N} \Theta_{M}{ }^{\alpha} \Theta_{N} \beta=$ $2 \Theta_{\Lambda}{ }^{[\alpha} \Theta^{\Lambda \beta]} \approx 0$. When the group $\mathrm{G}$ is not simple, then the indices $\alpha, \beta, \ldots$ refer only to the invariant subgroup that acts on the vector fields. This shows that the charges induced by the gauging must be mutually local, meaning that there exists an electric/magnetic duality transformation such that all the non-trivial gauge charges become electric. In the remainder of the text we will assume that we are dealing with a simple symmetry group $\mathrm{G}$, both for convenience and because this reflects the situation encountered in the maximal supergravity theories.

Let us continue to derive the $p$-form hierarchy for this specific example. First of all, one replaces the electric field strengths $F_{\mu \nu} \Lambda=2 \partial_{[\mu} A_{\nu]}{ }^{\Lambda}$ in the original ungauged Lagrangian by the electric components of the modified field strengths (2.17), which in the case at hand are written as,

$$
\mathcal{H}_{\mu \nu}{ }^{M}=\mathcal{F}_{\mu \nu}{ }^{M}+g Z^{M, \alpha} B_{\mu \nu \alpha}
$$

Here we used the definition

$$
B_{\mu \nu \alpha}=d_{\alpha M N} B_{\mu \nu}^{M N}
$$

Furthermore one replaces the ordinary derivatives (on the matter fields) by covariant ones, as specified in (4.12). Finally one adds a universal set of terms to the Lagrangian, which generalize (4.16). The Lagrangian thus takes the following form [3],

$$
\mathcal{L}_{\text {total }}=\mathcal{L}_{0}+\mathcal{L}_{\text {top }}
$$

where $\mathcal{L}_{0}$ is the original (ungauged) Lagrangian with the field strengths $F_{\mu \nu}{ }^{\Lambda}$ replaced by covariant field strengths $\mathcal{H}_{\mu \nu} \Lambda$, and the space-time derivatives $\partial_{\mu}$ by covariant derivatives $D_{\mu}$. The term $\mathcal{L}_{\text {top }}$ is the generalization of (4.16), and reads,

$$
\begin{aligned}
\mathcal{L}_{\text {top }}= & \frac{1}{8} g \varepsilon^{\mu \nu \rho \sigma} \Theta^{\Lambda \alpha} B_{\mu \nu \alpha}\left(2 \partial_{\rho} A_{\sigma \Lambda}+g X_{M N \Lambda} A_{\rho}{ }^{M} A_{\sigma}{ }^{N}-\frac{1}{4} g \Theta_{\Lambda}{ }^{\beta} B_{\rho \sigma \beta}\right) \\
& +\frac{1}{3} g \varepsilon^{\mu \nu \rho \sigma} X_{M N \Lambda} A_{\mu}{ }^{M} A_{\nu}{ }^{N}\left(\partial_{\rho} A_{\sigma}{ }^{\Lambda}+\frac{1}{4} g X_{P Q}{ }^{\Lambda} A_{\rho}{ }^{P} A_{\sigma}{ }^{Q}\right) \\
& +\frac{1}{6} g \varepsilon^{\mu \nu \rho \sigma} X_{M N}{ }^{\Lambda} A_{\mu}{ }^{M} A_{\nu}{ }^{N}\left(\partial_{\rho} A_{\sigma \Lambda}+\frac{1}{4} g X_{P Q \Lambda} A_{\rho}{ }^{P} A_{\sigma} Q\right) .
\end{aligned}
$$

The combined Lagrangian (4.25) is gauge invariant provided the embedding tensor $\Theta_{M}{ }^{\alpha}$ is constant and satisfies the closure constraint (2.7). The gauge transformations for the 1- and 2-form gauge fields have already been defined earlier in the context of an 
abstract $p$-form hierarchy, but as invariance of the Lagrangian they acquire a different form $[3,34]$,

$$
\begin{aligned}
\delta A_{\mu}{ }^{M}= & D_{\mu} \Lambda^{M}-g Z^{M, \alpha} \Xi_{\mu \alpha}, \\
\delta B_{\mu \nu \alpha}= & 2 D_{[\mu} \Xi_{\nu] \alpha}+2 d_{\alpha M N} A_{[\mu}{ }^{M} \delta A_{\nu]}{ }^{N}-2 d_{\alpha M N} \mathcal{G}_{\mu \nu}{ }^{M} \Lambda^{N} \\
& -g Y_{\alpha, M}{ }^{\beta} \Phi_{\mu \nu}{ }^{M}{ }_{\beta},
\end{aligned}
$$

where

$$
\mathcal{G}_{\mu \nu \Lambda}=\varepsilon_{\mu \nu \rho \sigma} \frac{\partial \mathcal{L}_{0}}{\partial \mathcal{H}_{\rho \sigma} \Lambda},
$$

is the covariant version of (4.2) and $\Xi_{\mu \alpha}=d_{\alpha M N} \Xi_{\mu}{ }^{M N}$. The covariant derivative of the transformation parameter $\Xi_{\mu \alpha}$ equals $D_{\mu} \Xi_{\nu \alpha}=\partial_{\mu} \Xi_{v \alpha}-g A_{\mu}{ }^{M} X_{M \alpha}{ }^{\beta} \Xi_{v \beta}$ with $X_{M \alpha}{ }^{\beta}=-\Theta_{M}{ }^{\gamma} f_{\gamma \alpha}{ }^{\beta}$ the gauge group generator in the adjoint representation of G. Observe that we have also included a 3-form gauge transformations with parameter $\Phi_{\mu \nu}{ }^{M}{ }_{\beta}$ in (4.27). As long as the closure constraint is satisfied, this transformation is irrelevant, since the 2-form field appears in the Lagrangian multiplied with $Z^{M, \alpha}$, which vanishes upon contraction with $Y_{\alpha, M}{ }^{\beta}$ by virtue of the closure constraint (c.f. (3.9)). This is the reason why the $p$-form hierarchy is truncated at $p=2$.

For what follows, it is convenient to present alternative expressions for the intertwining tensors $[6,15]$,

$$
\begin{aligned}
& Y_{\alpha, M}{ }^{\beta}=t_{\alpha M^{N}} \Theta_{N}{ }^{\beta}-X_{M}{ }^{\beta}{ }_{\alpha}, \\
& Y^{M}{ }_{\alpha, P} Q^{\beta}=-\delta_{P}{ }^{M} Y_{\alpha, Q^{\beta}}-\left(X_{P}\right)_{Q}{ }^{\beta, M}{ }_{\alpha}, \\
& Y^{M N}{ }_{\alpha, P Q R}^{\beta}=-\delta_{P}{ }^{M} Y^{N}{ }_{\alpha, Q R}^{\beta}-\left(X_{P}\right)_{Q R}{ }^{\beta, M N}{ }_{\alpha} \text {, }
\end{aligned}
$$

where the last terms $\left(X_{M}\right)$ denote the generators in the representation conjugate to the representations associated with the $p=2,3,4$ form fields. The above expressions are useful when performing explicit calculations. Note that all intertwining tensors are linear in the embedding tensor as well as in the generators $\left(t_{\alpha}\right)_{M}{ }^{N}$ or in the structure constants $f_{\alpha \beta} \gamma$. As was emphasized previously these tensors do not cover all the (irreducible) representations that are allowed by their index structure. For instance, the fact that the representation constraint (4.18) remains zero under the action of the rigid symmetry group, i.e. $\delta_{\alpha}\left(X_{(M N} Q \Omega_{P) Q}\right)=0$, implies that the following contraction

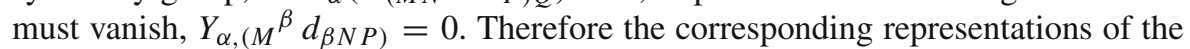
3-form field proportional to $\delta^{M}{ }_{(N} d_{\alpha P Q)}$ times a symmetric three-rank tensor will decouple from the hierarchy $[2,6]$.

It is possible to go beyond the $p=2$ truncation and introduce a 3 - and a 4-form field by making use of the observations at the end of Sect. 3.1. Hence we introduce a 3-form field $C_{\mu \nu \rho}{ }^{M}{ }_{\alpha}$ and a 4-form field $D_{\mu \nu \rho \sigma}{ }^{M N_{\alpha}}{ }_{\alpha}$. At the same time we relax the constraints on the embedding tensor, which we allow to be a space-time dependent field that transforms in the representation allowed by the representation constraint (4.18), but which will not be subject to the closure constraint (2.7). The 3- and 4-form fields 
then play the role of Lagrange multipliers that impose the constancy of the embedding tensor and the closure constraint. Now the Lagrangian takes the form

$$
\mathcal{L}_{\text {total }}=\mathcal{L}_{0}+\mathcal{L}_{\text {top }}+\mathcal{L}_{\mathrm{C}}
$$

where the first two terms are as before and the third term coincides with (3.12) applied to this particular case,

$$
\mathcal{L}_{C}=-\frac{1}{48} \varepsilon^{\mu \nu \rho \sigma}\left\{4 g C_{\nu \rho \sigma}{ }^{M}{ }_{\alpha} D_{\mu} \Theta_{M}{ }^{\alpha}+g^{2} D_{\mu \nu \rho \sigma}{ }^{M N}{ }_{\alpha} \mathcal{Q}_{M N}{ }^{\alpha}\right\} .
$$

Since the first two terms in (4.30) are only gauge invariant for a constant embedding tensor satisfying the closure constraint, there will be new variations proportional to $D_{\mu} \Theta_{M}{ }^{\alpha}$ or $\mathcal{Q}_{M N}{ }^{\alpha}$, which must be absorbed by the variations of (4.31). This requirement fixes the gauge transformation laws of the 3-and 4-form gauge fields. Note that the sub-representation in the 3-form field proportional to $\delta^{M}{ }_{(N} d_{\alpha P Q)}$ decouples from the Lagrangian, in view of the identity $D_{\mu} \Theta_{\left(M^{\alpha} d_{\alpha N P)}\right.}=0$. This is in accord with our discussion below (4.29).

The calculation of these variations is tedious but straightforward. A brief perusal of the variations shows that in the variation of $\mathcal{L}_{0}$, these terms originate from new variations of the covariant field strengths and the covariant derivatives. To see this we first note that the formal closure of the gauge algebra is affected,

$$
\left[X_{M}, X_{N}\right]=-X_{M N}^{P} X_{P}+\mathcal{Q}_{M N}^{\alpha} t_{\alpha}
$$

Furthermore the Ricci identity (2.20) is modified,

$$
\begin{aligned}
{\left[D_{\mu}, D_{\nu}\right]=} & -g \mathcal{F}_{\mu \nu}{ }^{M} X_{M}+\left[2 g A_{[\mu}{ }^{M} D_{\nu]} \Theta_{M}{ }^{\alpha}-g^{2} A_{[\mu}{ }^{M} A_{\nu]}{ }^{N} \mathcal{Q}_{M N}{ }^{\alpha}\right] t_{\alpha} \\
= & -g \mathcal{H}_{\mu \nu}{ }^{M} X_{M} \\
& +\left[2 g A_{[\mu}{ }^{M} D_{\nu]} \Theta_{M}{ }^{\alpha}-g^{2}\left(A_{[\mu}{ }^{M} A_{\nu]}{ }^{N}-B_{\mu \nu}{ }^{M N}\right) \mathcal{Q}_{M N}{ }^{\alpha}\right] t_{\alpha} .
\end{aligned}
$$

The transformation of the field strengths $\mathcal{H}_{\mu \nu}{ }^{M}$ will therefore become more complicated. Using (4.27) one finds the following result,

$$
\delta \mathcal{H}_{\mu \nu}{ }^{M}=-g \Lambda^{N} X_{N P}{ }^{M} \mathcal{G}_{\mu \nu}{ }^{P}-g \Lambda^{N} X_{P N}{ }^{M}(\mathcal{G}-\mathcal{H})_{\mu \nu}{ }^{P}+\Delta \mathcal{H}_{\mu \nu}{ }^{M},
$$

where $\Delta \mathcal{H}_{\mu \nu}{ }^{M}$ contains the new variations proportional to $D_{\mu} \Theta_{M}{ }^{\alpha}$ or $\mathcal{Q}_{M N}{ }^{\alpha}$. These take the form,

$$
\begin{aligned}
\Delta \mathcal{H}_{\mu \nu}{ }^{M}= & -2 g A_{[\mu}{ }^{N} D_{\nu]} \Theta_{N}{ }^{\alpha} t_{\alpha P}{ }^{M} \Lambda^{P}+g \Xi_{[\mu \alpha} D_{\nu]} \Theta_{N}{ }^{\alpha} \Omega^{M N} \\
& +g^{2} A_{[\mu}{ }^{N} A_{\nu]}{ }^{P} \Lambda^{Q}\left(\mathcal{Q}_{N P}{ }^{\alpha} t_{\alpha} Q^{M}+\mathcal{Q}_{Q N}{ }^{\alpha} t_{\alpha P}{ }^{M}\right) \\
& -\frac{1}{2} g^{2} \Lambda^{P} \mathcal{Q}_{N P}{ }^{\alpha} \Omega^{M N} B_{\mu \nu \alpha}-\frac{1}{2} g^{2} \Phi_{\mu \nu}{ }^{N}{ }_{\alpha} \Omega^{M P} \mathcal{Q}_{P N}{ }^{\alpha} .
\end{aligned}
$$


All these extra terms arise from the fact that the closure constraint no longer holds, and that the embedding tensor and related quantities are not constant and not gauge invariant anymore.

A similar result exists for the variation of a covariant derivative on a field transforming according to some representation of the gauge group,

$$
\delta\left(D_{\mu} \Phi\right)=g \Lambda^{M} X_{M} D_{\mu} \Phi+\Delta\left(D_{\mu}\right) \Phi
$$

where the second term is again proportional to $D_{\mu} \Theta_{M}{ }^{\alpha}$ or $\mathcal{Q}_{M N}{ }^{\alpha}$. This term takes the form,

$$
\Delta\left(D_{\mu}\right)=\left(g \Lambda^{M} D_{\mu} \Theta_{M}{ }^{\alpha}-g^{2} \Lambda^{M} A_{\mu}{ }^{N} \mathcal{Q}_{M N}{ }^{\alpha}+g^{2} \Xi_{\mu}{ }^{M N} \mathcal{Q}_{(M N)}{ }^{\alpha}\right) t_{\alpha} .
$$

The effect of the new variations of the field strengths and covariant derivatives thus lead to a new variation of the Lagrangian $\mathcal{L}_{0}$,

$$
\Delta \mathcal{L}_{0}=-\frac{1}{4} \varepsilon^{\mu \nu \rho \sigma} \mathcal{G}_{\rho \sigma \Lambda} \Delta \mathcal{H}_{\mu \nu}{ }^{\Lambda}+\frac{\delta \mathcal{L}_{0}}{\delta\left(D_{\mu} \Phi\right)} \Delta D_{\mu} \Phi
$$

What remains is to also evaluate the extra variations of the Lagrangian $\mathcal{L}_{\text {top }}$ defined in (4.26), which are also proportional to $D_{\mu} \Theta_{M}{ }^{\alpha}$ or $\mathcal{Q}_{M N}{ }^{\alpha}$. All these variations must then cancel against the variations of (4.31), provided one assigns the following gauge transformations to the 3-and 4-form gauge fields (for conciseness, we suppress the contributions coming from the second term in (4.38)),

$$
\begin{aligned}
\delta C_{\mu \nu \rho}{ }^{M}{ }_{\alpha}= & 3 D_{[\mu} \Phi_{\nu \rho]}{ }^{M}{ }_{\alpha}-3 A_{[\mu}{ }^{M} \delta B_{\nu \rho] \alpha}+3 \mathcal{G}_{[\mu \nu}{ }^{M} \Xi_{\rho] \alpha} \\
& +2 d_{\alpha P Q} A_{[\mu}{ }^{M} A_{\nu}{ }^{P} \delta A_{\rho]} Q-g Y^{M}{ }_{\alpha, P Q}{ }^{\beta} \Upsilon_{\mu \nu \rho} P Q_{\beta}, \\
\delta D_{\mu \nu \rho \sigma}{ }^{M N}{ }_{\alpha}= & 4 D_{[\mu} \Upsilon_{\nu \rho \sigma]}{ }^{M N}{ }_{\alpha}+\Lambda^{M} \mathcal{H}_{\mu \nu \rho \sigma}{ }^{N}+3\left(B_{[\mu \nu}{ }^{(M N)}-2 A_{[\mu}{ }^{M} A_{\nu}{ }^{N}\right) \delta B_{\rho \sigma] \alpha} \\
& +6 \mathcal{G}_{[\mu \nu}{ }^{M} \Phi_{\rho \sigma]}{ }^{N}{ }_{\alpha}+2 d_{\alpha P Q} A_{[\mu}{ }^{M} A_{\nu}{ }^{N} A_{\rho}{ }^{P} \delta A_{\sigma]} Q \\
& +4 \delta A_{[\mu}{ }^{M} C_{\nu \rho \sigma]}{ }^{N}{ }_{\alpha}-g Y^{M N}{ }_{\alpha, P Q R}{ }^{\beta} \Pi_{\mu \nu \rho \sigma}{ }^{P Q R}{ }_{\beta} .
\end{aligned}
$$

Here, the transformations parameterized by the functions $\Upsilon_{\mu \nu \rho}{ }^{M N}{ }_{\alpha}$ and $\Pi_{\mu \nu \rho \sigma}{ }^{M N P}{ }_{\alpha}$ are associated with the tensor gauge transformations of the 4- and 5-form fields. Of course, 5-form fields do not exist in a four-dimensional space-time, but this transformation still has some effect as it acts by a shift transformation on the 4-form field. The invariance of the Lagrangian under this transformation is ensured by the identity (3.10). Furthermore, $\mathcal{H}_{\mu \nu \rho \sigma}{ }^{M}{ }_{\alpha}$ is the covariant field strength associated with the 3-form field, 


$$
\begin{aligned}
& \mathcal{H}_{\mu \nu \rho \sigma}{ }^{M}{ }_{\alpha} \\
& =4 D_{[\mu} C_{\nu \rho \sigma]}{ }^{M}{ }_{\alpha}+12 d_{\alpha P Q} A_{[\mu}{ }^{M} A_{\nu}{ }^{P}\left(\mathcal{G}_{\rho \sigma]} Q_{-}-\frac{2}{3} \partial_{\rho} A_{\sigma]} Q_{-}-\frac{1}{2} g X_{R S} Q_{A_{\rho}}{ }^{R} A_{\sigma]} S\right) \\
& \quad-3 B_{[\mu \nu \alpha}\left(2(\mathcal{G}-\mathcal{H})_{\rho \sigma]}{ }^{M}+g Z^{M, \beta} B_{\rho \sigma] \beta}\right)+4 g Y_{\alpha, P}{ }^{\beta} A_{[\mu}{ }^{M} C_{\nu \rho \sigma]}{ }^{P} \\
& \quad+g Y^{M}{ }_{\alpha, P Q}{ }^{\beta} D_{\mu \nu \rho \sigma}{ }^{P Q}{ }_{\beta} .
\end{aligned}
$$

In defining this field strength we made use of our earlier observation that certain sub-representations of the 3-form field decouple from the theory.

At this point we have established the invariance of the Lagrangian (4.30). Rather than giving further calculational details which will be published elsewhere, we close with a number of comments. First of all, we have already observed that the $p$-form transformations obtained from the invariance of a certain Lagrangian, differ from the transformations that are obtained along the lines presented in Sect. 2.2. Nevertheless there exists a relation between these two sets of transformation rules. Namely they tend to be identical up to (Hodge) duality relations between $p$-forms, some of which are satisfied as a result of the field equations. However, as we know from [6], this relationship is only partially realized and there exist some unexpected invariances in Lagrangians such as (4.30) that are necessary for obtaining a consistent interpretation. This can be seen, in principle, by evaluating the commutator algebra based on the theory above, which will close up to these additional transformations.

Another intriguing feature of our result, which was noticed also in [6], is that for Lagrangians quadratic in derivatives, one can, in principle, integrate out the embedding tensor field $\Theta_{M}{ }^{\alpha}$. Although the resulting Lagrangian tends to be complicated and non-polynomial, it should encode all possible gaugings of this type. Whether or not this intriguing observation has any practical importance remains to be seen. We hope to return to these and related questions in the future.

Acknowledgments We are grateful to Hermann Nicolai and H. Samtleben for discussions. The work of M.v.Z. is part of the research program of the 'Stichting voor Fundamenteel Onderzoek der Materie (FOM)', which is financially supported by the 'Nederlandse Organisatie voor Wetenschappelijk Onderzoek (NWO)'. This work is also supported by NWO grant 047017015 .

Open Access This article is distributed under the terms of the Creative Commons Attribution Noncommercial License which permits any noncommercial use, distribution, and reproduction in any medium, provided the original author(s) and source are credited.

Note added in proof We include two recent papers on the $p$-form hierarchy in four space-time dimensions. The first one relates a modification of the representation constraint (4.18) to anomaly cancellation [37]. The second one considers the extension of the hierarchy with 3- and 4-form fields, and is directly related to the material presented in Section 4 [38].

\section{References}

1. de Wit, B., Samtleben, H., Trigiante, M.: The maximal $D=5$ supergravities, Nucl. Phys. B716, 215 (2005). hep-th/0412173

2. de Wit, B., Samtleben, H.: Gauged maximal supergravities and hierarchies of nonabelian vector-tensor systems. Fortsch. Phys. 53, 442 (2005). hep-th/0501243 
3. de Wit, B., Samtleben, H., Trigiante, M.: Magnetic charges in local field theory. JHEP 09, 016 (2005). hep-th/0507289

4. Nicolai, H., Samtleben, H.: Maximal gauged supergravity in three dimensions. Phys. Rev. Lett. 86, 1686 (2001). hep-th/0010076

5. Nicolai, H., Samtleben, H.: Compact and noncompact gauged maximal supergravities in three-dimensions. JHEP 0104, 022 (2001). hep-th/0103032

6. de Wit, B., Nicolai, H., Samtleben, H.: Gauged supergravities, tensor hierarchies, and M-theory. JHEP 02, 044 (2008). arXiv 0801.1294 [hep-th]

7. Cremmer, E., Julia, B., Scherk, J.: Supergravity theory in 11 dimensions. Phys. Lett. 76, 409 (1978)

8. Kaluza, Th.: Zum Unitätsproblem in der Physik. Sitzungsber.Preuss. Akad. Wiss. Berlin 1921, 966

9. Klein, O.: Quantentheorie und fünfdimensionale relativitätstheorie. Z. F. Physik 37, 895 (1926)

10. de Wit, B., Herger, I., Samtleben, H.: Gauged locally supersymmetric D = 3 nonlinear sigma models. Nucl. Phys. B671, 175 (2003). hep-th/0307006

11. de Wit, B., Nicolai, H., Samtleben, H.: Gauged supergravities in three dimensions: a panoramic overview. In: Proceedings of 27th Johns Hopkins Workshop on Current Problems in Particle Theory: Symmetries and Mysteries of M-Theory, Goteborg, Sweden, 24-26 Aug(2003). hep-th/0403014

12. Samtleben, H., Weidner, M.: The maximal $D=7$ supergravities. Nucl. Phys. B725, 383 (2005). hep-th/0506237

13. de Wit, B., Samtleben, H., Trigiante, M.: The maximal D = 4 supergravities. JHEP, 06, 049 (2007). arXiv:0705.2101 [hep-th]

14. Bergshoeff, E., Samtleben, H., Sezgin, E.: The gaugings of maximal D=6 supergravity. JHEP 03, 068 (2008). arXiv:0712.4277 [hep-th]

15. de Wit, B., Samtleben, H.: The end of the p-form hierarchy. JHEP 08, 015 (2008). arXiv 0805.4767 [hep-th]

16. Scherk, J., Schwarz, J.H.: How to get masses from extra dimensions. Nucl. Phys. B 153, 61-88 (1979)

17. Obers, N.A., Pioline, B.: U-duality and M-theory. Phys. Rept. 318, 113-225 (1999). hep-th/9809039

18. de Wit, B., Nicolai, H.: Hidden symmetries, central charges and all that. Class. Quant. Grav. 18, 3095-3112 (2001). hep-th/0011239

19. Elitzur, S., Giveon, A., Kutasov, D., Rabinovici, E.: Algebraic aspects of matrix theory on $T^{d}$. Nucl. Phys. B509, 122-144 (1998). hep-th/9707217

20. de Wit, B., Hoppe, J., Nicolai, H.: On the quantum mechanics of supermembranes. Nucl. Phys. B 305, 545 (1988)

21. Banks, T., Fischler, W., Shenker, S.H., Susskind, L.: M-Theory as a matrix model: a conjecture. Phys. Rev. D55, 5112 (1997). hep-th/9610043

22. Hull, C., Townsend, P.K.: Unity of superstring dualities. Nucl. Phys. B438, 109 (1995). hep-th/9410167

23. Iqbal, A., Neitzke, A., Vafa, C.: A mysterious duality. Adv. Theor. Math. Phys. 5, 769-808 (2002). hep-th/0111068

24. West, P.C.: E(11) origin of brane charges and U-duality multiplets. JHEP 08, 052 (2004). hep-th/0406150

25. Riccioni, F., West, P.: The E(11) origin of all maximal supergravities. JHEP 07, 063 (2007). arXiv:0705.0752 [hep-th]

26. Bergshoeff, E.A., De Baetselier, I., Nutma, T.A.: E(11) and the embedding tensor. JHEP 09, 047 (2007). arXiv:0705.1304 [hep-th]

27. Riccioni, F., West, P.: E(11)-extended spacetime and gauged supergravities. JHEP 0802, 039 (2008). arXiv:0712.1795 [hep-th]

28. Bergshoeff, E.A., Hohm, O., Nutma, T.A.: A note on $\mathrm{E}_{11}$ and three-dimensional gauged supergravity. JHEP 0805, 081 (2008). arXiv:0803.2989 [hep-th]

29. Englert, F., Houart, L., Kleinschmidt, A., Nicolai, H., Nassiba, T.: An E9 multiplet of BPS states. JHEP 05, 065 (2007). hep-th/0703285

30. Bergshoeff, E.A., Hohm, O., Kleinschmidt, A., Nicolai, H., Nutma, T.A., Palmkvist, J.: E 10 and gauged maximal supergravity. arXiv:0810.5767 [hep-th]

31. Samtleben, H., Weidner, M.: Gauging hidden symmetries in two dimensions. JHEP 08, 076 (2007). arXiv:0705.2606 [hep-th]

32. Schön, J., Weidner, M.: Gauged $N=4$ supergravities. JHEP 05, 034 (2006). hep-th/0602024

33. Derendinger, J.P., Petropoulos, P.M., Prezas, N.: Axionic symmetry gaugings in $N=4$ supergravities and their higher-dimensional origin. Nucl. Phys. B785, 115 (2007). arXiv:0705.0008 [hep-th]

34. de Vroome, M., de Wit, B.: Lagrangians with electric and magnetic charges in $N=2$ supersymmetric gauge theories. JHEP 08, 064 (2007). arXiv:0707.2717 [hep-th] 
35. de Wit, B.: Electric-magnetic duality in supergravity. Nucl. Phys. Proc. Suppl. 101, 154 (2001). hepth/0103086

36. de Wit, B., Lauwers, P.G., Van Proeyen, A.: Lagrangians of $N=2$ supergravity-matter systems. Nucl. Phys. B 255, 569 (1985)

37. De Rydt, J., Schmidt, T.T., Trigiante, M., Van Proeyen, A., Zagermann, M.: Electric/magnetic duality for chiral gauge theories with anomaly cancellation. JHEP 12, 105 (2008). arXiv:0808.2130[hep-th]

38. Bergshoeff, E.A., Hartong, J., Hohm, O., Huebscher, M., Ortin, T.: Gauge theories, duality relations and the tensor hierarchy, arXiv:0901.2054[hep-th] 